\title{
Paclitaxel-mediated human aryl hydrocarbon receptor mRNA translation by an internal ribosomal entry site-dependent mechanism
}

\author{
WEN-QING GAO*, JING MA*, LIU-LIU SUN, QI LI, RUI-YU ZHU and JIAN JIN \\ Laboratory of Molecular Pharmacology, School of Pharmaceutical Sciences, \\ Jiangnan University, Wuxi, Jiangsu 214122, P.R. China
}

Received November 18, 2016; Accepted May 2, 2017

DOI: $10.3892 /$ or.2017.5958

\begin{abstract}
The aryl hydrocarbon receptor (AHR) is a ligandactivated transcription factor that is best known in mediating the toxicities of dioxins and dioxin-like compounds. AHR is activated by a variety of endogenous ligands and participating in tumor development. Thus, it will provide a new approach for cancer prevention and treatment to study the translation mechanism of AHR in tumor cells. In this study, we show that the 5'-untranslated region (UTR) of AHR mRNA contains an internal ribosome entry site (IRES). After mapping the entire AHR 5'-UTR, we determined that the full-length 5'-UTR is indispensable for the highest IRES activity. Interestingly, we found that AHR expression is induced in ovarian (A2780), breast (MDA-MB231), hepatic (Bel7402) and colorectal cancer cells (SW620) by chemotherapeutic drug paclitaxel (PTX) through IRES-dependent translation mechanism. Moreover, IRES activity is increased in the PTX-resistant ovarian cancer cells in which AHR protein expression was also enhanced. These results strongly suggest an important role for AHR IRES-dependent translation mechanism in cancer cell response to paclitaxel treatment.
\end{abstract}

\section{Introduction}

The aryl hydrocarbon receptor (AHR) is a ligand-activated transcription factor that is best known to mediate the toxicities of dioxins and dioxin-like compounds (1). After ligand binding, AHR translocates to the nucleus and dimerizes

Correspondence to: Dr Rui-Yu Zhu or Professor Jian Jin, Laboratory of Molecular Pharmacology, School of Pharmaceutical Sciences, Jiangnan University, 1800 Lihu Road, Wuxi, Jiangsu 214122, P.R. China

E-mail: ry_zhu@sina.com

E-mail: jinjian31@hotmail.com

${ }^{*}$ Contributed equally

Key words: aryl hydrocarbon receptor, ribosome entry site, translation, paclitaxel, drug-resistance with the aryl hydrocarbon nuclear translocator (ARNT) and activates the expression of a battery of genes containing specific DNA-enhancer sequences, which are known as xenobiotic-responsive elements (XREs) (2). These genes encode xenobiotic-metabolizing enzymes, which are involved in biotransformation and cellular detoxification process $(3,4)$. Phenotype of AHR-null mice showed developmental defects in the hepatic, hematopoietic, cardiovascular and immune systems (5-9). As such, AHR pathway is believed to contribute significantly to sense the environmental chemicals. In addition to cellular detoxification, AHR also regulates signal transduction pathways involved in cellular melabolism, proliferation, differentiation, and apoptosis (10). Several studies have shown that AHR, which is expressed at aberrantly high levels in a wide panel of tumors, plays a critical role in tumor progression by enhancing tumor invasion and migration $(11,12)$. Increasing expression of AHR and its binding partner, ARNT, has been noted in hepatocellular carcinoma (HCC) (13). Furthermore, recent evidence suggests that the possibility that AHR contributes to cell invasion and migration through upregulation of stem cell (e.g., Notch1,2, Sox2 and Pou5F1/Oct4) and invasion/migration-associated genes (e.g., Snail1, Snail2, Twist1,2, NDRG1 and p53) (14-16).

It has been reported that ligand-induced expression of AHR occurs at the transcriptional level. So far, no report exists on the regulation of AHR expression at the translational level $(17,18)$. Translation initiation of mRNAs in eukaryotic cells is a complex process and affects the overall rate of protein synthesis (19). For most mRNAs, the global translation initiation is mediated by ribosome scanning whereby the translation initiation complexes recognizes and binds to the cap structure at the 5'-end of mRNA and scans the mRNA in 5'-3' direction until the first AUG codon within an optimal context is encountered. The global translation initiation was inhibited by a variety of conditions including nutrient deprivation, heat shock, apoptosis and viral infection. Yet, despite this occurrence, a minority of cellular mRNA can still be translated by internal ribosome entry sites (IRES)-dependent translation initiation $(20,21)$. IRES elements were first characterized in picornaviruses that contain long 5'-UTRs that naturally lack 5'-terminal cap structures $(22,23)$. IRES elements were also discovered in 
several mammalian mRNAs encoding regulatory proteins induced in growth control and programmed cell death. Fibroblast growth factor 2 (FGF2), insulin-like growth factor II (IGF2), the protooncogene c-myc, NF- $\kappa$ B repressing factor (NRF), X-linked inhibitor of apoptosis protein (XIAP), heat shock protein 70 (Hsp70) and Aurora A kinase, have all been shown to contain IRES (24-30). Since many transcription factors contain IRES, it was hypothesized the AHR mRNA would also contain an IRES. In the present study, evidence is provided demonstrating that the 5'-UTR of AHR mRNA contains an IRES. Importantly, overexpression of AHR through IRES-dependent translation initiation contributes to cancer cells survival under drug stress. Therefore, these results imply that translation initiation by internal ribosome entry may represent an important mechanism for the regulation of AHR expression in cancer cells.

\section{Materials and methods}

Construction of dicistronic reporter plasmids. The initial dicistronic vector pRF containing the reporter genes for Renilla luciferase (first cistron, RL) and firefly luciferase (second cistron, FL) was described previously $(31,32)$. To obtain plasmids pR-NRF-F and pR-AHR-F, we inserted the 5'-UTRs of AHR and NRF which were synthesized by Sangon Biotech. Co. (Shanghai, China) into pRF respectively. pBR-AHR-F was constructed by deleting SV40 promoter from pR-AHR-F and used to analyze the cryptic promoter of AHR 5'-UTR. To detect the core region for IRES activity, AHR 5'-UTR was deleted to different length sequences by PCR. The primers with $E c o$ RI and NdeI restriction sites are listed in Table I. PCRs were performed using PrimerSTAR Max DNA polymerase (Takara, Dalian, China) according to the manufacturer's instructions and the resulting products were inserted into pRF. All PCRs were conducted at $98^{\circ} \mathrm{C}$ for $3 \mathrm{~min}$ followed by 32 cycles of $98^{\circ} \mathrm{C}$ for $10 \mathrm{sec}, 68^{\circ} \mathrm{C}$ for $20 \mathrm{sec}$ and $72^{\circ} \mathrm{C}$ for $1 \mathrm{~min}$, and $72^{\circ} \mathrm{C}$ for $5 \mathrm{~min}$.

Cell culture and treatment. All cell lines were obtained from American Type Culture Collection. Cell lines HCT-8, Bel7402 and A2780/PTX were maintained in RPMI-1640 medium (Gibco; Thermo Fisher Scientific), where other cell lines were cultured in Dulbecco's modified Eagle's medium (Gibco; Thermo Fisher Scientific). A2780/PTX supplemented with $0.3 \mathrm{mg} / \mathrm{ml}$ PTX and all cell lines were maintained in medium supplemented with $10 \%$ fetal bovine serum (FBS) at $37^{\circ} \mathrm{C}$, $5 \% \mathrm{CO}_{2}$. For drug treatment, A2780, MB231, Bel7302 and SW620 cell lines were cultured in medium supplemented with clinically relevant concentration of PTX $(0.2$ and $0.4 \mu \mathrm{g} / \mathrm{ml})$ and without PTX for $24 \mathrm{~h}$. PTX was purchased from Sigma.

Luciferase assays and transient transfection. Cells were grown to 60-70\% confluency in 24-well plate and transfected with $1 \mu \mathrm{g}$ of plasmid DNA using $2 \mu \mathrm{l}$ Lipofectamine $^{\mathrm{TM}} 2000$ (Invitrogen, Thermo Fisher Scientific, Inc.) per well, according to the manufacturer's instructions. Cells were harvested after incubation for $24 \mathrm{~h}$ at $37^{\circ} \mathrm{C}$. Renilla and firefly luciferase activities were quantitated using the Dual-luciferase reporter system (Promega Corp.) and a GloMAX ${ }^{\mathrm{TM}} 2020$ luminometer. Cell lysate $(20 \mu \mathrm{l})$ was combined sequentially with firefly then
Renilla luciferase substrates (100 $\mu \mathrm{l})$ according to the manufacturer's instructions. Light emission was measured $3 \mathrm{sec}$ after addition of each of the substrates and integrated over a 10 -sec interval. These experiments were repeated more than three times.

SiRNA transfection. RNAi duplexes for AHR and siNC (negative control) duplexes were purchased from Santa Cruz Biotechnology (sc-29654; sc-37007). Cells were seeded in RPMI-1640 or DMEM containing 10\% FBS and maintained for $24 \mathrm{~h}$. The cells were transfected with siRNA or siNC using Lipofectamine 2000 (Invitrogen, Thermo Fisher Scientific) according to the manufacturer's protocol.

MTT assay. A2780, A549, MB231, Bel7402, HCT-8, SW620 and HEK293 cells were seeded at a density of 3,000 cells per well containing $100 \mu \mathrm{l}$ of medium in a 96-well plate for $24 \mathrm{~h}$, then transfected with 10 pmol siNC or siAHR using $1 \mu \mathrm{l}$ Lipofectamine 2000 (Invitrogen, Thermo Fisher Scientific) per well. After $72 \mathrm{~h}$, the surviving cell number was determined as described previously (33).

Reverse transcriptase (RT)-PCR and quantitative (q) PCR. Total RNA was isolated from treated cells using TRIzol reagent (Invitrogen) and was reverse transcribed using a reserves transcription system (Takara) to generate a cDNA template according to the manufacturer's instructions. RT-PCR was performed with PrimerSTAR Max DNA polymerase (Takara) according to the manufacturer's instructions. The PCR was conducted at $98^{\circ} \mathrm{C}$ for $3 \mathrm{~min}$, followed by 32 cycles of $98^{\circ} \mathrm{C}$ for $10 \mathrm{sec}, 68^{\circ} \mathrm{C}$ for $50 \mathrm{sec}$, and $72^{\circ} \mathrm{C}$ for $5 \mathrm{~min}$. Amplification products were visualized on a $1 \%$ agarose gel staining by ethidium bromide under UV light and quantified by ImageJ software (NIH, Bethesda, MD, USA). qRCR was performed with SYBR ${ }^{\circledR}$ Premix Ex Taq ${ }^{\mathrm{TM}}$ (Takara) according to the manufacturer's instructions and quantified with a Roach Lightcycler 480 . The PCR was conducted at $95^{\circ} \mathrm{C}$ for $10 \mathrm{~min}$, followed by 40 cycles of $95^{\circ} \mathrm{C}$ for $10 \mathrm{sec}, 60^{\circ} \mathrm{C}$ for $30 \mathrm{sec}$, and $95^{\circ} \mathrm{C}$ for $10 \mathrm{sec}, 60^{\circ} \mathrm{C}$ for $60 \mathrm{sec}$ and $95^{\circ} \mathrm{C}$ for $15 \mathrm{sec}$. The primers were: for R1 (41-210), forward, 5'-TGATAACTGGTC CGCAGTGGT-3' and reverse, 5'-TACTGGCTCAATATGTG GCACAA-3'; for R2 (701-865), forward, 5'-TGCTTATCTAC GTGCAAGTGATG-3' and reverse, 5'-CCCATTTCATCAGG TGCATCTT-3'; for F1 (69-340) forward, 5'-CGAGCAGCTG CACAAAGCCAT-3' and reverse, 5'-GCTCGCGCTCGTTGT AGATG-3'; for AHR 5'-UTR, forward, 5'-AGTGGCTGGGG AGTCCCGTC-3' and reverse, 5'-GGTGCCCAGCCGACG GCG-3'; for AHR, forward, 5'-CCCATATCCGAATGATTA AGACTG-3' and reverse, 5'-CGTAAATGCTCTGTTCCT TCC-3; for $\beta$-actin, forward, 5'-TGAAGTGTGACGTGGAC ATC-3' and reverse, 5'-GGAGGAGCAATGATCTTGAT-3'.

Protein analysis. Western blot analyses were performed using standard procedures. Cells were harvested in cell lysis buffer RIPA (Roche) and phosphatase inhibitors (Pierce). Cell extracts were separated by $12 \%$ SDS-PAGE and transferred to polyvinylidene difluoride (PVDF) membranes. The membranes were incubated with $5 \%$ milk in Tris-buffered saline (TBS) overnight at $4^{\circ} \mathrm{C}$. The blots were washed three times in TBS, incubated with polyclonal antibodies directed against AHR 
Table 1. Oligonucleotide primers used in PCR for plasmids construct.

No.

Sequences of oligonucleotide primers 5'-3'

\begin{tabular}{ll}
-518 to -1 & F: GGAATTCCATATGGCTCAGAACAGGGGCAGCCGTG \\
-493 to -1 & R: CGGAATTCGGTGCCCAGCCGACGGCG \\
-404 to -1 & F: GGAATTCCATATGCCCGAGCTCCGCAGGCGG \\
& R: CGGAATTCGGTGCCCAGCCGACGGCG \\
-313 to -1 & F: GGAATTCCATATGCGGTCACGGGGCGCGGC \\
-212 to -1 & R: CGGAATTCGGTGCCCAGCCGACGGCG \\
-613 to -212 & F: GGAATTCCATATGGCGGGCATTGCCGCGCCG \\
-613 to -313 & R: CGGAATTCGGTGCCCAGCCGACGGCG \\
& F: GGAATTCCATATGAGCTCACCTGTACTGGCGCGGG \\
-613 to -493 & R: CGGAATTCGGTGCCCAGCCGACGGCG \\
-613 to -518 & F: GGAATTCCATATGAGTGGCTGGGGAGTCCCGTCGA \\
& R: CGGAATTCTGCCTGGGCCTGGCGCAGTGA \\
& F: GGAATTCCATATGAGTGGCTGGGGAGTCCCGTCGA \\
& R: CGGAATTCCAGGTGAGGCGGCCCGGG \\
& F: GGAATTCCATATGAGTGGCTGGGGAGTCCCGTCGA \\
& R: CGGAATTCGCGCGGGCCACCAGTCCC \\
\hline
\end{tabular}

F, forward; R, reverse.

(1:1,000; sc-74571; Santa Cruz Biotechnology), caspase-3 (1:500; ab32042; Abcam), survivin (1:5,000; ab76424; Abcam) and $\beta$-actin (1:1,000; AA128; Beyotime Institute of Biotechnology) for $4 \mathrm{~h}$ at room temperature, washed, incubated with secondary antibodies respectively (1:1,000; A0216; Beyotime Institute of Biotechnology) for $2 \mathrm{~h}$, washed. Then proteins were detected by a chemiluminescent detection system (Tanton, Shanghai, China) and quantified by ImageJ software (NIH).

Statistical analysis. All experiments were performed at least three times. The data were analyzed using Graphpad Prism 5.0 (La Jolla, CA, USA) and presented as mean values \pm SEM. Statistical analysis was performed using a t-test. $\mathrm{p}<0.05$ was set as the statistically significant difference.

\section{Results}

AHR knockdown inhibits the growth of cancer cells. AHR is highly expressed in a wide panel of tumors and previous studies indicated that overexpression of AHR could help cancer cells survive under cellular stress $(34,35)$. To address whether AHR imparts survival to cancer cells, AHR was knocked down in a variety of cell lines including human ovarian cancer cell line (A2780), human lung cancer cell line (A549), human breast cancer cell line (MDA-MB231), human hepatocellular carcinoma cell line (Bel7402), human colon cancer cell line (HCT-8 and SW620) and human embryonic kidney 293 cells
(HEK293). After transfection with siNC or siAHR for $72 \mathrm{~h}$, the protein expression of AHR was examined in each cell line via western blotting (Fig. 1A). The results showed that the levels of AHR from each cell line in the siAHR group were significantly reduced compared to the siNC group. In addition, knockdown of AHR expression by siRNA specific for human AHR resulted in an increased expression level of caspase-3 and a decreased expression level of survivin (a member of the inhibitor of apoptosis family), especially in A549 and HCT-8 cell lines, indicating that the critical role of AHR for the anti-apoptotic effect. Next, a dimethylthiazolyl diphenyl tetrazolium (MTT) assay was performed and showed that the reduction of AHR decreased the growth of HEK293 and multiple cancer cell lines, especially in A2780 and A549 cell lines (2.5- and 2.4-fold). These data reveal that knocking down AHR could suppress the proliferation of various cancer cells. Therefore, overexpression of AHR may facilitate the growth of cancer cells.

Characterization of the 5'-UTR of AHR $m R N A$. This study aimed to identify elements that regulate AHR translation. After searching the NCBI database, we found that AHR mRNA has an usually long 5'-UTR (613 bases), which may present the activity of the IRES (Fig. 2A). In addition, this sequence had an average predicted folding energy $(\Delta \mathrm{G}=-321.48 \mathrm{kcal} / \mathrm{mole})$ and contained eight distinct stem-loop structures, which are often detected in RNAs known to initiate translation via IRES (Fig. 2B). Then, RT-PCR assays were carried out 


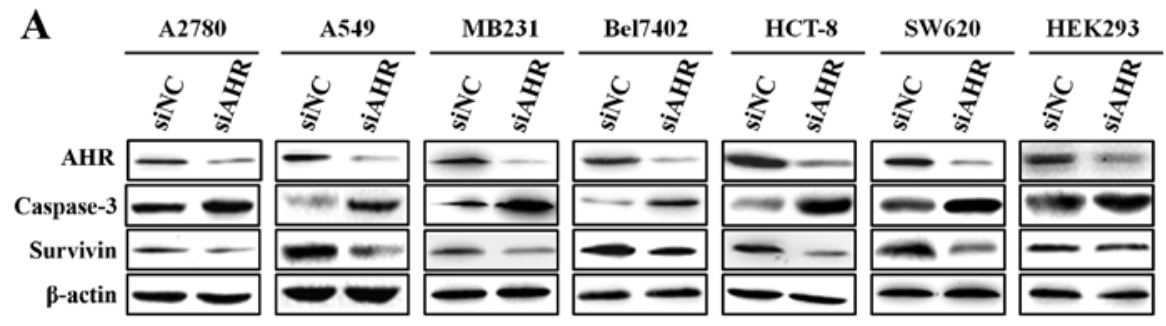

B

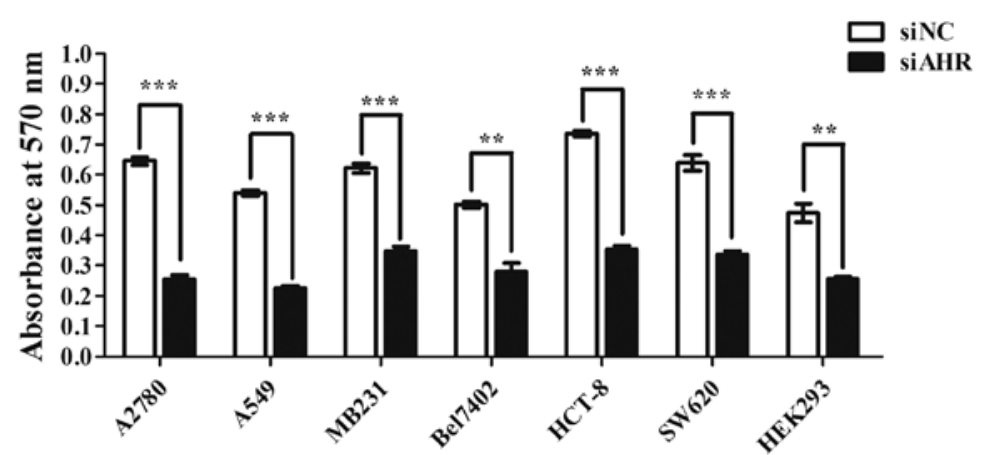

Figure 1. AHR knockdown inhibits the growth of cancer cells. (A) A2780, A549, MB231, Bel7402, HCT-8, SW620 and HEK293 cell lines were transfected with siNC (control) or siAHR, respectively. The AHR, caspase-3 and survivin protein levels from each cell line were analyzed $72 \mathrm{~h}$ post-transfection by western blotting. $\beta$-actin served as a loading control. (B) MTT assays show the proliferation of A2780, A549, MB231, Bel7402, HCT-8, SW620 and HEK293 cell lines after transfection with siRNA against AHR or control siRNA. Data represent the mean \pm SEM of three independent experiments. ${ }^{* *} \mathrm{p}<0.001 ;{ }^{* * * *} \mathrm{p}<0.0001$.

$\mathbf{A}$

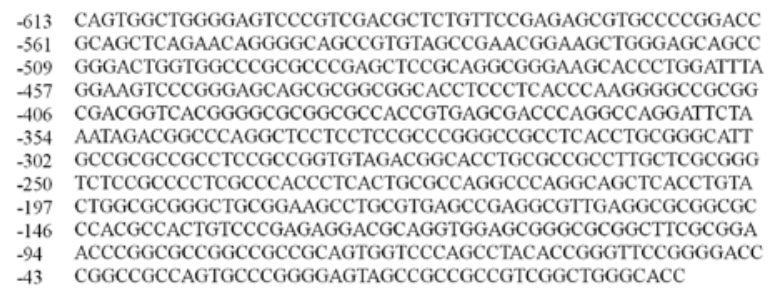

B

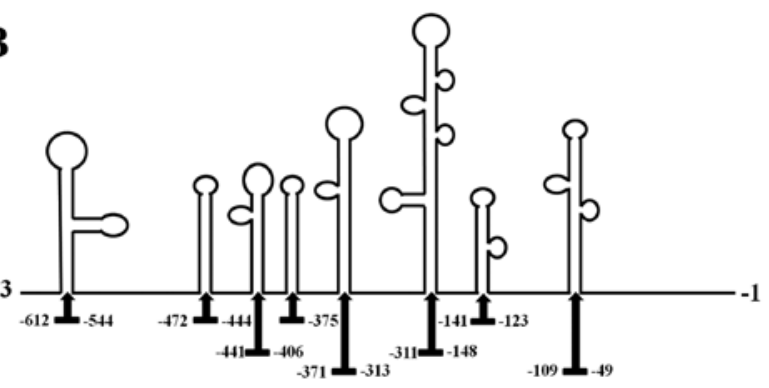

C

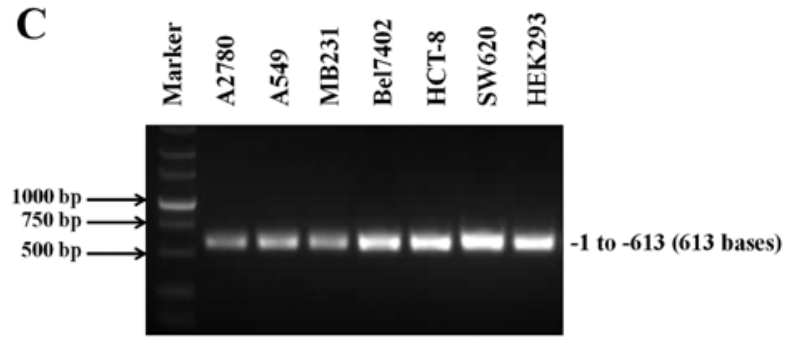

Figure 2. Characterization of the 5'-UTR of AHR mRNA. (A) DNA sequence of the AHR 5'-UTR (NM_001314027.1). (B) Predicted RNA secondary structure of the 5'-UTR (nt -613 to -1). The folded structures of individual portions of the UTR were generated using mFold software (v2.3). (C) RT-PCR products indicate that AHR 5'-UTR was expressed in HEK293 and in all cancer cells studied (A2780, A549, MB231, Bel7402, HCT-8 and SW620). to analyze the expression of AHR 5'-UTR in HEK293 and various cancer cell lines (A2780, A549, MB231, Bel7402, HCT-8 and SW620). The results demonstrated and confirmed the presence of the 5'-UTR of AHR mRNA in HEK293 and all cancer cells studied (Fig. 2C).

Analysis of IRES activity in the 5'-UTR of AHR mRNA. To determine if the AHR 5'-UTR contains an IRES, we employed the dicistronic reporter plasmid pRF (31). The dicistronic construct contains a Renilla luciferase (RL) open reading frame (ORF) and a firefly luciferase (FL) ORF that are separated by an intercistronic region. Translation of the Renilla cistron occurs by a cap-dependent mechanism, while translation of the second cistron will not occur unless there is an IRES present to initiate translation. Accordingly, the AHR 5'-UTR was inserted into the intercistronic region (Fig. 3A). The 5'-UTR of the NRF mRNA, which is known to harbor a strong IRES element, was utilized as a positive control (30). HEK293 was transfected with the dicistronic constructs and both luciferase activities were measured. As predicted, the firefly luciferase activity was markedly increased by the insertion of the AHR 5'-UTR. An approximately 30 -fold increase was observed in the pR-AHR-F construct. AHR 5'-UTR was also 3.2 times better at promoting IRES-mediated translation than the well-characterized IRES in NRF mRNA, the positive control. To ensure that the increase in firefly luciferase activity was a result of enhanced translation rather than the presence of cryptic promoter activity in AHR 5'-UTR sequence, a promoterless plasmid pBR-AHR-F was constructed. HEK293 cells were transfected with pR-AHR-F and pBR-AHR-F plasmids, respectively, and luciferase activity was measured. No activity of Renilla and firefly luciferase was detected in cells transfected with pBR-AHR-F (Fig. 3B). 
A

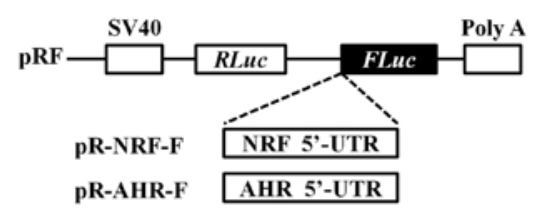

B

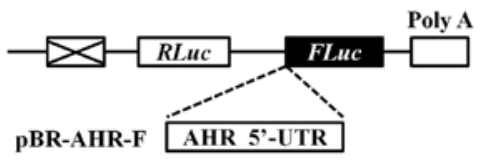

C

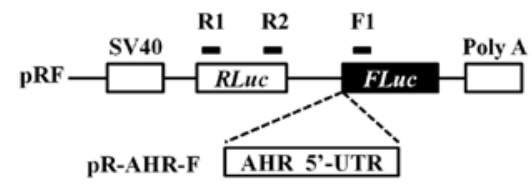

HEK293
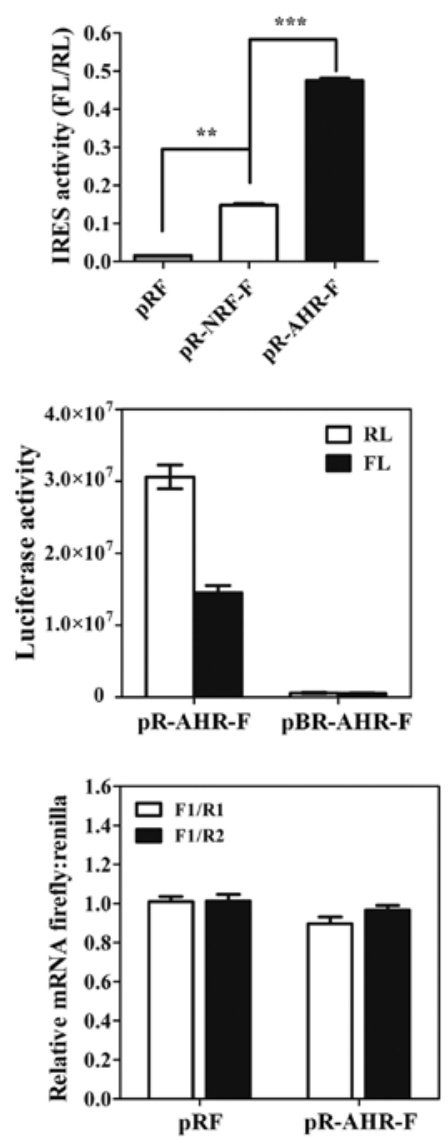

D

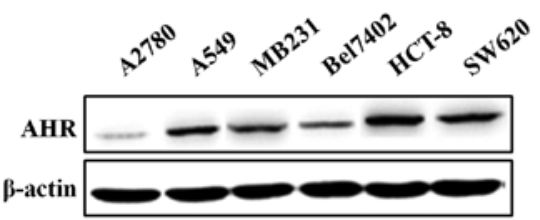

$\mathbf{E}$

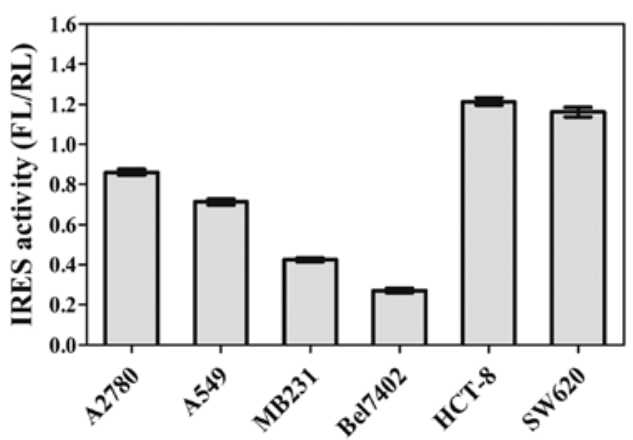

Figure 3. The AHR 5'-UTR exhibits IRES activity. (A) The dicistronic plasmids pRF, pR-NRF-F and pR-AHR-F were transfected into HEK293 cells, $24 \mathrm{~h}$ after transfection, the cells were lysed and luciferase activity was measured respectively. The ratio of FL to RL represents IRES activity. ${ }^{* *} \mathrm{p}<0.001 ;{ }^{* * *} \mathrm{p}<0.0001$. (B) Analysis of the cryptic promoter activity in the AHR 5'-UTR element. The vector pR-AHR-F and the promoterless vector pBR-AHR-F were used for reporter assays. Both luciferase activities were determined following transfection in HEK293 cells. (C) Transcriptional level ratio of firefly and Renilla influenced by IRES activity of AHR 5'-UTR sequence. (D) Endogenous expressions of AHR in A2780, A549, MB231, Bel7402, HCT-8 and SW620 cell lines. (E) The dicistronic plasmid pR-AHR-F was transfected into each cell line respectively. The ratio of FL to RL represents IRES activity. Data represent the mean \pm SEM of three independent experiments.

The production of functional monocistronic firefly luciferase mRNAs may be caused by alternative splicing events that removed the Renilla ORF. Therefore, reporter mRNA of firefly and Renilla was analyzed by qRT-PCR to obtain the ratio of FL and RL mRNA. The ratio from intact dicistronic message is $1: 1$ such as pRF in Fig. $3 \mathrm{C}$ and the existence of splicing site results a ratio higher than 1 . After analyzing the detected sequence AHR and control, the ratio of each was lower than 1 , indicating that there was no evidence for a cryptic splicing event that result in loss of the Renilla cistron. Taken together, these results suggested that an effective IRES is present in AHR 5'-UTR sequence. To determine whether the relative 
A

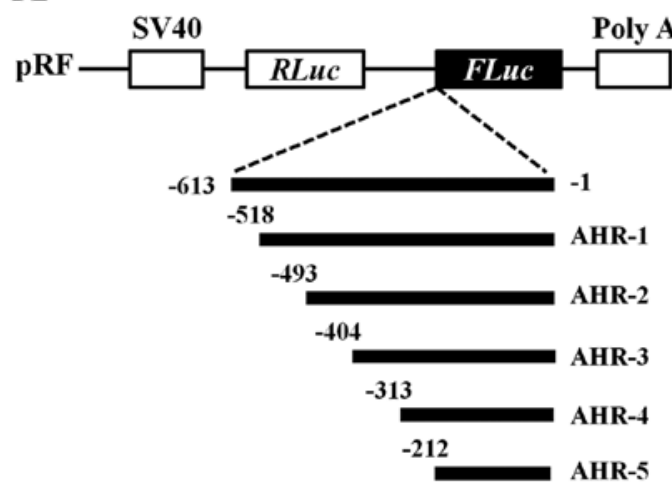

C

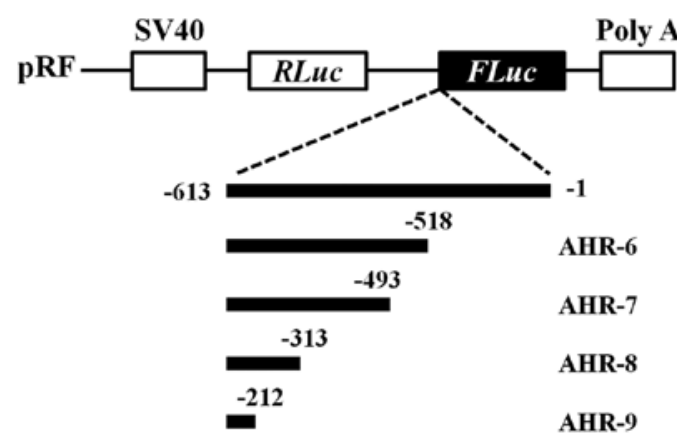

B

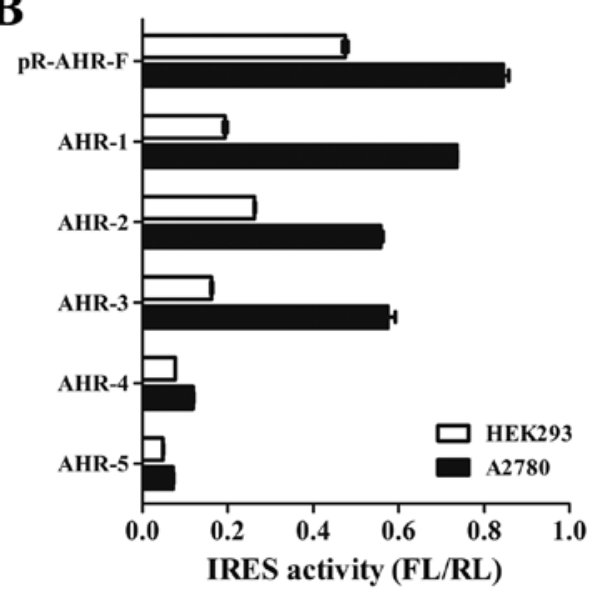

D

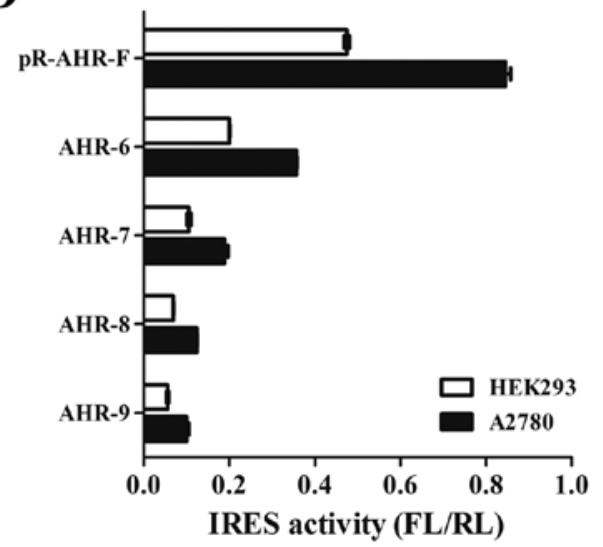

Figure 4. Mapping the AHR IRES. (A) Schematic representation of the 5' deleted regions of the AHR 5'-UTR and these regions were inserted into pRF between Renilla and firefly luciferase reporter genes. (B) IRES activity of different truncated AHR sequences in HEK293 and A2780 cell lines. (C) Schematic representation of the 3 ' deleted regions of the AHR 5'-UTR and these regions were inserted into pRF as described in A. (D) IRES activity of different truncated AHR sequences in HEK293 and A2780 cell lines. The expression data are presented as the mean \pm SEM of triplicate samples.

activity of the AHR IRES is dependent upon the cell type, we tested AHR expression levels and IRES activities in 6 cancer cell lines. The results shown in Fig. 3D and E demonstrate that AHR IRES activity parallels AHR protein expression in all cancer cell lines except for A2780 cells, indicating that IRES-dependent translation may be contributing to the overexpression of the AHR protein observed in the subset of cancer cell lines.

Mapping the AHR IRES. To further determine the position of the IRES within the AHR 5'-UTR, a series of deletions from the 5 '-end of the UTR to -518 (-518 to -1$),-493(-493$ to -1$),-404$ (-404 to -1$),-313(-313$ to -1$)$ and $-212(-212$ to -1) was cloned into the dicistronic vector (Fig. 4A). The plasmids were transfected into HEK293 and A2780 cells and the relative luciferase activity was determined for each of these truncations. As seen in Fig. 4B, deletion of the 5'-end $209 \mathrm{nt}$ resulted in little change in IRES activity (cf. -518 to $-1,-493$ to -1 and -404 to -1 ) in A2780 cells. Conversely, truncation of the IRES element from -404 to -313 or -212 nucleotides diminished IRES activity to $<20 \%$, suggesting that the region between -404 and -313 is required for maximal IRES activity. To establish a 3 ' border, deletions from the 3'-end were tested in the dicistronic vector: -613 to $-212,-613$ to -313 . -613 to -493 and -613 to -518 (Fig. 4C). At the 3 '-end, deletion of $211 \mathrm{nt}(-613$ to -212$)$ resulted in 2.3-fold decrease in downstream cistron activity in HEK293 and A2780 cells (Fig. 4D). Further three mutations approximately abolish their translation initiation activity (cf. -613 to $-313,-613$ to -493 and -613 to -518). The deletion analysis indicated that sequences at both the $5^{\prime}$ and $3^{\prime}$ ends of the AHR are essential for the integrity of the IRES.

AHR is overexpressed by IRES mechanism after PTX treatment. Cellular IRES is generally used under pathophysiological conditions when cap-dependent scanning is compromised, such as cellular stress and apoptosis. In order to determine whether AHR 5'-UTR regulates its protein expression in cancer cells after PTX treatment, the endogenous expression of AHR was first measured by western blotting. A2780, MB231, Bel7402 and SW620 cells were treated with increasing concentrations $(0.2$ and $0.4 \mu \mathrm{g} / \mathrm{ml})$ of PTX for $24 \mathrm{~h}$ and AHR protein expression was examined. As shown in Fig. 5A, AHR expression was significantly increased in each cell line treated with PTX. Moreover, we observed that the PTX-induced AHR expression was dose-dependent. To evaluate whether the induction of AHR occurred at transcriptional level, we performed qRT-PCR to examine AHR 
A
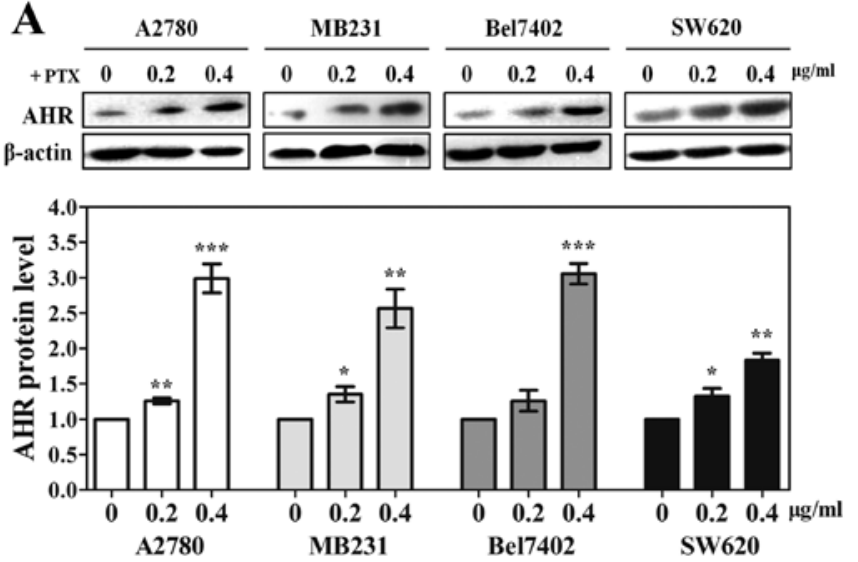

B

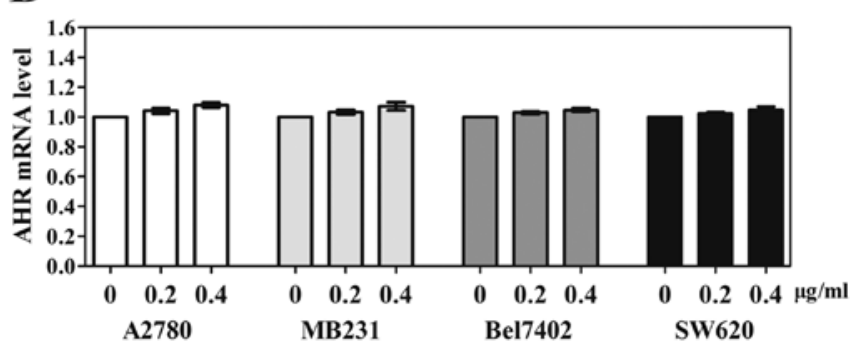

C

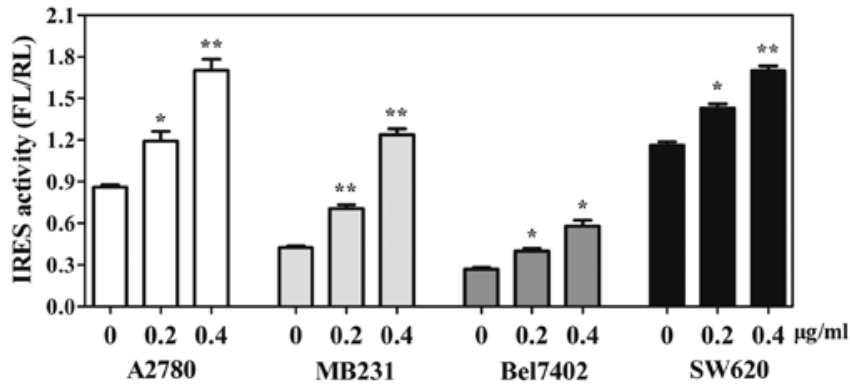

Figure 5. AHR is overexpressed by IRES mechanism after PTX treatment (A) A2780, MB231, Bel7402 and SW620 cell lines were treated with and without PTX $(0.2,0.4 \mu \mathrm{g} / \mathrm{ml})$ respectively, for $24 \mathrm{~h}$. The AHR protein expression was examined by western blot analysis and normalized to $\beta$-actin. The AHR/ $\beta$-actin ratio from treated group was compared with the level from untreated group in each cell line, which was set to $1 .{ }^{*} \mathrm{p}<0.05 ;{ }^{* *} \mathrm{p}<0.001$ **** $\mathrm{p}<0.0001$. (B) mRNA expression level of AHR after treatment with or without PTX in each cell line, as determined by qRT-PCR. The mRNA level from treated group was compared with the level from untreated group in each cell line, which was set to 1. (C) The construct pR-AHR-F was transfected into A2780, MB231, Bel7402 and SW620 cell lines treated with and withou PTX respectively. FL and RL activity were determined and the IRES activity is shown. The expression data are presented as the mean \pm SEM of triplicate samples. ${ }^{*} \mathrm{p}<0.05 ;{ }^{* *} \mathrm{p}<0.001 ;$ Student's t-test.

mRNA expression in each cell line. We found that PTX had no effect on AHR transcription in any of the cancer cell lines (Fig. 5B). These results indicated the elevated AHR may be translated by IRES-dependent mechanism. Next, we performed gene transfections and reporter assays to measure the IRES activity of AHR 5'-UTR in each cell line with PTX treatment. As expected, following with the elevation of the dose, the inducible IRES activity was obviously increased in all cell lines, especially in MB231 cells (Fig. 5C). Taken together, these data suggest that IRES-dependent translation mechanism plays a critical role in tumor cell adaptation to PTX treatment.
A
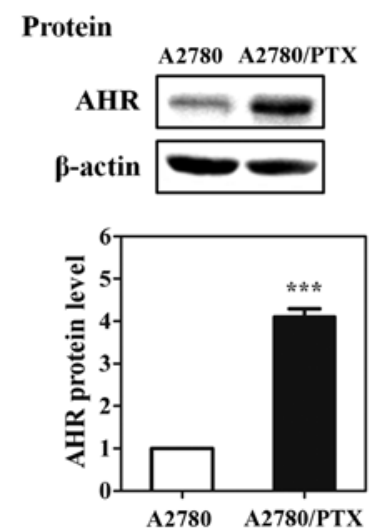

C

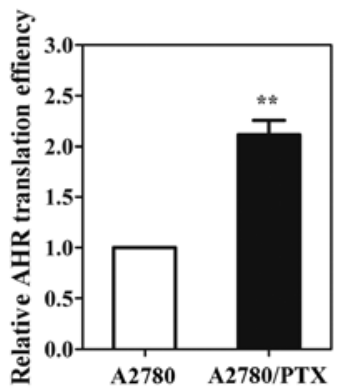

B

RNA
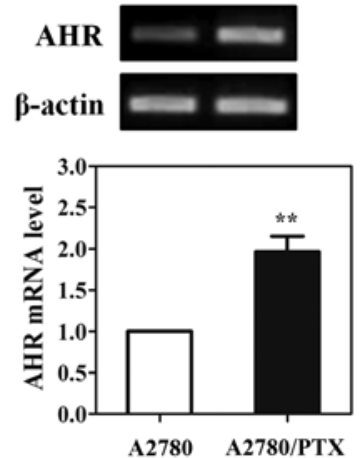

D

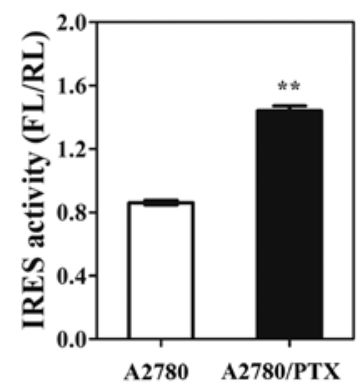

Figure 6. IRES activity is increased in PTX-resistant ovarian cancer cells. (A) AHR protein levels were analyzed in A2780 and A2780/PTX cell lines by western blotting and normalized to $\beta$-actin. The AHR/ $\beta$-actin ratio from A2780/PTX cells was compared with the level from A2780 cells, which was set to $1{ }^{* * *}$ p $<0.0001$. (B) mRNA expression level of AHR in each cell line was determined by RT-PCR. The mRNA level from A2780/PTX was compared with the level from A2780, which was set to $1 .{ }^{* *} \mathrm{p}<0.001$. (C) The relative translational efficiency of AHR was shown as ratio of AHR protein/mRNA. Data represent the mean \pm SEM of three independent experiments. ${ }^{* *} \mathrm{p}<0.001$. (D) The IRES activities of AHR 5' leader were examined by dual-luciferase reporter assay in wild-type and PTX-resistant ovarian cancer cell lines. Data represent the mean \pm SEM of three independent experiments. ${ }^{* *} \mathrm{p}<0.001$.

IRES activity is increased in PTX-resistant ovarian cancer cells. In this study, the AHR protein levels were enhanced in various wild-type cancer cells after treating with PTX. To study whether AHR was overexpressed in drug-resistant cancer cells, we measured the endogenous protein and mRNA levels of AHR in PTX-resistant and wild-type ovarian cancer cell lines, respectively. We found that AHR protein expression level was significantly increased in A2780/PTX cells (Fig. 6A). In addition, there was also an increase in AHR mRNA level in the drug-resistant cells compared to the wild-type cells (Fig. 6B). To test whether the translational efficacy was increased in A2780/PTX cells, MDR1 protein level was normalized to the mRNA level in each cell line (Fig. 6C). Interestingly, the protein-to-mRNA level was 2.1-fold higher in A2780/PTX cells, indicating that the elevated protein synthesis contributed to the enhanced AHR protein in A2780/PTX cells. To detect AHR IRES activity in drug-resistant cancer cells, we performed the dual-luciferase assay in PTX-resistant A2780 cells. Results, as shown in Fig. 6D, demonstrated that the AHR 5'-UTR had more efficiently IRES activity ( 1.67-fold higher) in PTX-resistant ovarian cancer cells. Therefore, we proposed that the increased IRES activity may promote the 
AHR protein production in drug-resistant ovarian cancer cells.

\section{Discussion}

AHR is present in a variety of organisms and has important function in both physiological and toxicological processes. At the cellular level, AHR involves in governing cell proliferation and cell cycle, cell morphology, cell adhesion and cell migration (36). Moreover, in ovarian cancer, it has been demonstrated that ITE regulates cancer cell proliferation and migration via AHR (37). In human pluripotent embryonic teratocarcinoma NTERA2 cells, it has been proven that Alu retrotransposons promote cell differentiation through AHR (36). Also, in breast cancer cells, overexpression of AHR facilitated cell proliferation and migration via upregulation of NDRG1 under hypoxia (15). Similarly, our study indicated that knocking down AHR was able to decrease cell proliferation in human ovarian, lung, breast, hepatocellular and colon cancer cells (Fig. 1).

As AHR is highly expressed in a wide panel of tumors, molecules with antagonistic AHR activity could be considered potential candidates for the treatment of such diseases. The results of the present study provide evidence that AHR expression can also be regulated at the initiation stage of translation through an IRES located within the 5'-UTR of its mRNA. An IRES is experimentally defined as a sequence element that can facilitate the initiation of translation of a downstream cistron in a dicistronic mRNA. By this assay, we demonstrated that AHR 5'-UTR has significant IRES activity with the luminescence ratio 3.2-fold higher than the activity of positive control (NRF) in HEK293 cells (Fig. 3). To investigate the function of AHR IRES element in cancer cells, we performed gene transfections and reporter assays in seven cancer cell types which were categorized as high or low AHR protein expressing cells (Fig. 1A). We found that AHR IRES activity correlated with AHR protein expression in most cancer cell lines, suggesting that IRES-dependent translation initiation mediated by the AHR 5'-UTR is used to a greater extent in cells overexpressing the AHR protein.

Based on our deletion analysis, it appears that the full-length 5'-UTR of AHR mRNA exhibited the maximal IRES activity. However, deletion of sequence between -404 and -303 or between -212 and -1 produces a remarkable negative effect on the IRES activity of AHR mRNA, indicating that these regions are important parts of a larger sequence forming the IRES element of 5'-UTR of AHR mRNA (Fig. 4). The IRES-dependent translation requires the presence of an additional complex set of trans-acting factors (ITAFs) for translational activity to occur (38-41). We propose that the important regions of AHR 5'-UTR may bind to the trans-acting factors, inducing the conformational changes that facilitate recruitment of the ribosome to the AHR IRES.

Generally, IRES becomes activated under conditions in which cap-dependent protein synthesis is greatly reduced, whereupon the activated IRES initiates translation of only those specific proteins that are able to protect cells from stress. For instance, the IRES activity of TNF receptor-associated factor 1 (TRAF1) is simulated in leukemic cells treated with vincristine, the 5'-UTR of human sensitivity to nitrogen mustard (hSNM1) upregulates protein expression in HT-1080 cells during mitosis, and several apoptosis-inducing agents induce the IRES activity of cellular inhibitor of apoptosis protein 1 (c-IAP1) in 293T cells (42-44). Furthermore, previous studies have demonstrated that AHR nuclear translocator (ARNT isoform 1) impairs chemo-resistance and survival to cancer cells (45). Thus, the increasing concentration of chemotherapeutic drug PTX was used to treat A2780, MB231, Bel7402 and SW620 cell lines. Here, we observed that PTX induced AHR IRES activity, which plays a key regulatory role in controlling overexpressed AHR protein in each cell line (Fig. 5). Moreover, we found IRES-mediated translation mechanism contributing to enhanced AHR protein expression in PTX-resistant ovarian cancer cells. Indeed, our results demonstrated that the AHR IRES-dependent mechanism provides the cancer cell with the plasticity needed to respond to rapid changes in the environment, such as chemotherapeutic drug pressure or apoptosis.

In conclusion, we demonstrated that the human AHR 5'-UTR contains a strong IRES regulating its translation. In an examination of various cell lines, IRES activity was a pivotal mechanism that correlated with AHR protein expression. Moreover, this mechanism seems to be upregulated during cellular stress, indicating that targeting this mechanism may be beneficial for treating multiple tumors.

\section{Acknowledgements}

This study was supported by the National Natural Science Foundation of China (81101667) and the Natural Science Foundation of Jiangsu Province (BK2009071).

\section{References}

1. Tian J, Feng Y, Fu H, Xie HQ, Jiang JX and Zhao B: The aryl hydrocarbon receptor: A key bridging molecule of external and internal chemical signals. Environ Sci Technol 49: 9518-9531, 2015.

2. Beischlag TV, Luis Morales J, Hollingshead BD and Perdew GH: The aryl hydrocarbon receptor complex and the control of gene expression. Crit Rev Eukaryot Gene Expr 18: 207-250, 2008.

3. Safe S and Krishnan V: Cellular and molecular biology of aryl hydrocarbon $(\mathrm{Ah})$ receptor-mediated gene expression. Arch Toxicol (Suppl) 17: 99-115, 1995.

4. Hanieh H: Toward understanding the role of aryl hydrocarbon receptor in the immune system: Current progress and future trends. BioMed Res Int 2014: 520763, 2014.

5. Gonzalez FJ and Fernandez-Salguero P: The aryl hydrocarbon receptor: Studies using the AHR-null mice. Drug Metab Dispos 26: 1194-1198, 1998.

6. Schmidt JV, Su GH, Reddy JK, Simon MC and Bradfield CA: Characterization of a murine Ahr null allele: Involvement of the Ah receptor in hepatic growth and development. Proc Natl Acad Sci USA 93: 6731-6736, 1996.

7. Lahvis GP, Lindell SL, Thomas RS, McCuskey RS, Murphy C, Glover E, Bentz M, Southard J and Bradfield CA: Portosystemic shunting and persistent fetal vascular structures in aryl hydrocarbon receptor-deficient mice. Proc Natl Acad Sci USA 97: 10442-10447, 2000.

8. Lahvis GP, Pyzalski RW, Glover E, Pitot HC, McElwee MK and Bradfield CA: The aryl hydrocarbon receptor is required for developmental closure of the ductus venosus in the neonatal mouse. Mol Pharmacol 67: 714-720, 2005.

9. Gasiewicz TA, Singh KP and Casado FL: The aryl hydrocarbon receptor has an important role in the regulation of hematopoiesis: Implications for benzene-induced hematopoietic toxicity. Chem Biol Interact 184: 246-251, 2010. 
10. Mulero-Navarro S and Fernandez-Salguero PM: New trends in aryl hydrocarbon receptor biology. Front Cell Dev Biol 4: 45, 2016.

11. Salisbury TB and Tomblin JK: Insulin/insulin-like growth factors in cancer: New roles for the aryl hydrocarbon receptor, tumor resistance mechanisms, and new blocking strategies. Front Endocrinol (Lausanne) 6: 12, 2015.

12. Feng $S$, Cao Z and Wang X: Role of aryl hydrocarbon receptor in cancer. Biochim Biophys Acta 1836: 197-210, 2013.

13. Wang LT, Chiou SS, Chai CY, Hsi E, Wang SN, Huang SK and Hsu SH: Aryl hydrocarbon receptor regulates histone deacetylase 8 expression to repress tumor suppressive activity in hepatocellular carcinoma. Oncotarget 8: 7489-7501, 2017.

14. Stanford EA, Wang Z, Novikov O, Mulas F, Landesman-Bollag E, Monti S, Smith BW, Seldin DC, Murphy GJ and Sherr DH: The role of the aryl hydrocarbon receptor in the development of cells with the molecular and functional characteristics of cancer stem-like cells. BMC Biol 14: 20, 2016

15. Li EY, Huang WY, Chang YC, Tsai MH, Chuang EY, Kuok QY, Bai ST, Chao LY, Sher YP and Lai LC: Aryl hydrocarbon receptor activates NDRG1 transcription under hypoxia in breast cancer cells. Sci Rep 6: 20808, 2016.

16. Li ZD, Wang K, Yang XW, Zhuang ZG, Wang JJ and Tong XW: Expression of aryl hydrocarbon receptor in relation to p53 status and clinicopathological parameters in breast cancer. Int J Clin Exp Pathol 7: 7931-7937, 2014.

17. Chang CY and Puga A: Constitutive activation of the aromatic hydrocarbon receptor. Mol Cell Biol 18: 525-535, 1998.

18. Opitz CA, Litzenburger UM, Sahm F, Ott M, Tritschler I, Trump S, Schumacher T, Jestaedt L, Schrenk D, Weller M, et al: An endogenous tumour-promoting ligand of the human aryl hydrocarbon receptor. Nature 478: 197-203, 2011.

19. Hinnebusch AG and Lorsch JR: The mechanism of eukaryotic translation initiation: new insights and challenges. Cold Spring Harb Perspect Biol: July 18, 2012 (Epub ahead of print). doi: $10.1101 /$ cshperspect.a011544.

20. Shatsky IN, Dmitriev SE, Terenin IM and Andreev DE: Cap- and IRES-independent scanning mechanism of translation initiation as an alternative to the concept of cellular IRESs. Mol Cells 30: 285-293, 2010.

21. Komar AA and Hatzoglou M: Cellular IRES-mediated translation: The war of ITAFs in pathophysiological states. Cell Cycle 10: 229-240, 2011.

22. Jang SK, Kräusslich HG, Nicklin MJ, Duke GM, Palmenberg AC and Wimmer E: A segment of the 5' nontranslated region of encephalomyocarditis virus RNA directs internal entry of ribosomes during in vitro translation. J Virol 62: 2636-2643, 1988.

23. Pelletier $\mathbf{J}$ and Sonenberg N: Internal initiation of translation of eukaryotic mRNA directed by a sequence derived from poliovirus RNA. Nature 334: 320-325, 1988.

24. Holcik M, Lefebvre C, Yeh C, Chow T and Korneluk RG: A new internal-ribosome-entry-site motif potentiates XIAP-mediated cytoprotection. Nat Cell Biol 1: 190-192, 1999.

25. Subkhankulova T, Mitchell SA and Willis AE: Internal ribosome entry segment-mediated initiation of c-Myc protein synthesis following genotoxic stress. Biochem J 359: 183-192, 2001

26. Dobson T, Chen J and Krushel LA: Dysregulating IRESdependent translation contributes to overexpression of oncogenic Aurora A kinase. Mol Cancer Res 11: 887-900, 2013.

27. Gonzalez-Herrera IG, Prado-Lourenco L, Pileur F, Conte C, Morin A, Cabon F, Prats H, Vagner S, Bayard F, Audigier S, et al: Testosterone regulates FGF-2 expression during testis maturation by an IRES-dependent translational mechanism. FASEB J 20: 476-478, 2006

28. Pedersen SK, Christiansen J, Hansen T, Larsen MR and Nielsen FC: Human insulin-like growth factor II leader 2 mediates internal initiation of translation. Biochem J 363: 37-44, 2002.
29. Rubtsova MP, Sizova DV, Dmitriev SE, Ivanov DS, Prassolov VS and Shatsky IN: Distinctive properties of the 5'-untranslated region of human hsp70 mRNA. J Biol Chem 278: 22350-22356, 2003.

30. Oumard A, Hennecke M, Hauser $H$ and Nourbakhsh $M$ : Translation of NRF mRNA is mediated by highly efficient internal ribosome entry. Mol Cell Biol 20: 2755-2759, 2000.

31. Gao W, Li Q, Zhu R and Jin J: La autoantigen induces ribosome binding protein 1 (RRBP1) expression through internal ribosome entry site (IRES)-mediated translation during cellular stress condition. Int J Mol Sci 17: 1174, 2016.

32. Dai W, Ma W, Li Q, Tao Y, Ding P, Zhu R and Jin J: The 5'-UTR of DDB2 harbors an IRES element and upregulates translation during stress conditions. Gene 573: 57-63, 2015.

33. Zhang Y, Chao T, Li R, Liu W, Chen Y, Yan X, Gong Y, Yin B, Liu W, Qiang B, et al: MicroRNA-128 inhibits glioma cells proliferation by targeting transcription factor E2F3a. J Mol Med (Berl) 87: 43-51, 2009.

34. Fujisawa Y, Li W, Wu D, Wong P, Vogel C, Dong B, Kung HJ and Matsumura F: Ligand-independent activation of the arylhydrocarbon receptor by ETK (Bmx) tyrosine kinase helps MCF10AT1 breast cancer cells to survive in an apoptosis-inducing environment. Biol Chem 392: 897-908, 2011.

35. Yin J, Sheng B, Qiu Y, Yang K, Xiao W and Yang H: Role of AhR in positive regulation of cell proliferation and survival. Cell Prolif 49: 554-560, 2016.

36. Morales-Hernández A, González-Rico FJ, Román AC, Rico-Leo E, Alvarez-Barrientos A, Sánchez L, Macia Á, Heras SR, García-Pérez JL, Merino JM, et al: Alu retrotransposons promote differentiation of human carcinoma cells through the aryl hydrocarbon receptor. Nucleic Acids Res 44: 4665-4683, 2016.

37. Wang K, Li Y, Jiang YZ, Dai CF, Patankar MS, Song JS and Zheng J: An endogenous aryl hydrocarbon receptor ligand inhibits proliferation and migration of human ovarian cancer cells. Cancer Lett 340: 63-71, 2013.

38. Martínez-Salas E, Lozano G, Fernandez-Chamorro J, FranciscoVelilla R, Galan A and Diaz R: RNA-binding proteins impacting on internal initiation of translation. Int J Mol Sci 14: 21705-21726, 2013.

39. Costa-Mattioli M, Svitkin Y and Sonenberg N: La autoantigen is necessary for optimal function of the poliovirus and hepatitis $\mathrm{C}$ virus internal ribosome entry site in vivo and in vitro. Mol Cell Biol 24: 6861-6870, 2004.

40. Cobbold LC, Wilson LA, Sawicka K, King HA, Kondrashov AV, Spriggs KA, Bushell M and Willis AE: Upregulated c-myc expression in multiple myeloma by internal ribosome entry results from increased interactions with and expression of PTB-1 and YB-1. Oncogene 29: 2884-2891, 2010.

41. Cobbold LC, Spriggs KA, Haines SJ, Dobbyn HC, Hayes C, de Moor CH, Lilley KS, Bushell M and Willis AE: Identification of internal ribosome entry segment (IRES)-trans-acting factors for the Myc family of IRESs. Mol Cell Biol 28: 40-49, 2008.

42. Yang L, Gu L, Li Z and Zhou M: Translation of TRAF1 is regulated by IRES-dependent mechanism and stimulated by vincristine. Nucleic Acids Res 38: 4503-4513, 2010.

43. Zhang X, Richie C and Legerski RJ: Translation of hSNM1 is mediated by an internal ribosome entry site that upregulates expression during mitosis. DNA Repair (Amst) 1: 379-390, 2002.

44. Van Eden ME, Byrd MP, Sherrill KW and Lloyd RE: Translation of cellular inhibitor of apoptosis protein 1 (c-IAP1) mRNA is IRES mediated and regulated during cell stress. RNA 10: 469-481, 2004.

45. Gardella KA, Muro I, Fang G, Sarkar K, Mendez O and Wright CW: Aryl hydrocarbon receptor nuclear translocator (ARNT) isoforms control lymphoid cancer cell proliferation through differentially regulating tumor suppressor p53 activity. Oncotarget 7: 10710-10722, 2016. 